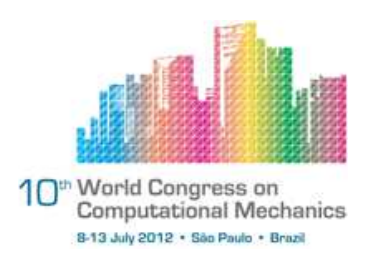

\title{
LARGE DEFLECTION OF COMPOSITE LAMINATE THIN PLATES BY THE BOUNDARY ELEMENT METHOD
}

\author{
L. C. Silveira ${ }^{1}$, E. L. Albuquerque ${ }^{1}$ \\ ${ }^{1}$ Department of Mechanical Engineering, Faculty of Technology at University of Bras Ãlia
}

\begin{abstract}
Boundary-integral equations for large deflections of thin plates are presented. Quadratic boundary elements are used to discretise the boundary. Domain integrals that arise from non-linear terms are transformed into boundary integrals using the radial integration method. As a result, the obtained formulation does not demand domain discretization. For the solution of the non-linear system, the total incremental method is used. A numerical example is presented and comparisons with other numerical results are made to demonstrate the accuracy of the proposed method. The formulation illustrates the adaptability of the boundary element methods to non-linear problems.
\end{abstract}

Keywords: Boundary Element Method, Radial Integration Method, Thin Plates.

\section{INTRODUCTION}

The boundary element method has already been established as an extremely efficient and effective technique in the analysis of linear and non-linear structural problems, as can be seen in the book of Aliabadi [1]. For linear problems, it is always possible to find a fundamental solution considering all terms of governing equations, producing formulations with pure boundary discretization. However, when non-linear differential equations are considered, fundamental solutions are found only to the linear portion of the equations. As a result, non-linear formulations have not only boundary integrals but also domain integrals. Thus, the advantage in reducing the dimensionality, as is the case in linear boundary element method, is lost in the non-linear case. Some procedures has been developed to avoid domain discretization, as can be seen in the works of Purbolaksono and Aliabadi [2] or Wen et al. [3,4]. Apart from numerous articles where the boundary element method is applied to non-linear isotropic problems, to the best of authors knowledge, it has not been found in literature a paper where the boundary element method is applied to a non-linear analysis of anisotropic materials.

The linear theory of Kirchhoff for thin plates is valid only when the transverse deflections are approximately less than the thickness of the plate. The extension to large deflections, where the non-linear terms are retained in the kinematic relationships, leads to a pair of coupled non-linear fourth order equations for the transverse displacement and the stress function for the in-plane stress resultants. Purbolaksono and Aliabadi [5] discuss and compare some approaches to compute large deflections of isotropic plates using the boundary 
element method. Non-linear formulations demand iterative solutions that, depending on the chosen approach, is necessary to rebuild influence matrices for each load step. This kind of procedure gives better results. However, they are very time consuming. This work presents a boundary element formulation for the analysis of large deflections in thin plates.

\section{BOUNDARY ELEMENT FORMULATION}

The hypothesis of large deflections used in this work is:

- Deflections $u_{3}$ (displacements in the direction of axis $x_{3}$, transversal to the plate) are large (many times bigger than the thickness $h$ of the plate) but strains are still small when compared to the unity (changes in geometry can be disregarded).

Membrane stress resultants $N_{i j}(i, j=1,2)$, i.e., stress due to extension, compression or shear on the medium plane of the laminate, integrated along the thickness, for a composite laminated plate, considering large deflections, are given by (Chia [7]):

$\left\{\begin{array}{l}N_{11} \\ N_{22} \\ N_{12}\end{array}\right\}=\left[\begin{array}{lll}A_{11} & A_{12} & A_{16} \\ A_{12} & A_{22} & A_{26} \\ A_{16} & A_{26} & A_{66}\end{array}\right]\left(\left\{\begin{array}{l}u_{1,1} \\ u_{2,2} \\ u_{1,2}\end{array}\right\}+\frac{1}{2}\left\{\begin{array}{c}u_{3,1}^{2} \\ u_{3,2}^{2} \\ 2 u_{3,1} u_{3,2}\end{array}\right\}\right)=\left\{\begin{array}{c}N_{11} \\ N_{22} \\ N_{12}\end{array}\right\}^{(l)}+\left\{\begin{array}{c}N_{11} \\ N_{22} \\ N_{12}\end{array}\right\}^{(n l)}$,

where:

$$
\left\{\begin{array}{l}
N_{11} \\
N_{22} \\
N_{12}
\end{array}\right\}^{(l)}=\left[\begin{array}{lll}
A_{11} & A_{12} & A_{16} \\
A_{12} & A_{22} & A_{26} \\
A_{16} & A_{26} & A_{66}
\end{array}\right]\left\{\begin{array}{l}
u_{1,1} \\
u_{2,2} \\
u_{1,2}
\end{array}\right\}
$$

and

$$
\left\{\begin{array}{l}
N_{11} \\
N_{22} \\
N_{12}
\end{array}\right\}=\left[\begin{array}{ccc}
A_{11} & A_{12} & A_{16} \\
A_{12} & A_{22} & A_{26} \\
A_{16} & A_{26} & A_{66}
\end{array}\right]\left\{\begin{array}{c}
u_{3,1}^{2} \\
u_{3,2}^{2} \\
2 u_{3,1} u_{3,2}
\end{array}\right\}
$$

indices $(l)$ and $(n l)$ stand for linear and non-linear components, respectively, $A_{i j}(i, j=$ $1,2,6)$ are terms of the laminate extension stiffness matrix; $u_{i}(i=1,2)$ are displacement in directions of axes $x_{1}$ and $x_{2}, u_{3}$ is the deflection, i.e., displacement in the direction $x_{3}$, normal to the plate surface.

In the absence of body forces, governing equations of an anisotropic plate under large deflections are written as (Shi [8]):

$$
\begin{aligned}
& N_{i j, j}=0, \\
& D_{11} u_{3,1111}+4 D_{16} u_{3,1112}+2\left(D_{12}+D_{66}\right) u_{3,1122}+4 D_{26} u_{3,1222}+D_{22} u_{3,2222}=N_{i j} u_{3, i j}
\end{aligned}
$$


where $i, j, k=1,2 ; D_{11}, D_{22}, D_{66}, D_{12}, D_{16}$, and $D_{26}$ are terms of the laminate bending stiffness matrix.

Integral equations for in-plane displacements, obtained by the reciprocity and Green theorems and equations (4) and (5), are given by (O'Donoghue e Atluri [6]):

$$
\begin{gathered}
c_{i j} u_{j}(Q)+\int_{\Gamma} t_{i j}^{*}(Q, P) u_{j}(P) d \Gamma(P)=\int_{\Gamma} u_{i j}^{*}(Q, P) t_{j}^{(l)}(P) d \Gamma(P) \\
+\int_{\Omega} N_{i j}^{(n l)}(P) u_{k i, j}^{*}(P, Q) d \Omega
\end{gathered}
$$

where $t_{i}^{(l)}=N_{i j}^{(l)} n_{j}$ stand for tractions on the boundary of the plate, parallel to the plane defined by $x_{1}-x_{2} ; n_{j}$ is the unity vector normal to the boundary at the source point $Q ; P$ is the field point; asterisks stands for fundamental solutions. Fundamental solutions of anisotropic plane elasticity can be found in Sollero and Aliabadi [9]. The constant $c_{i j}$ is introduced in order to take into account that the source point $Q$ can be in the domain, on the boundary, or outside the domain.

Resultant stresses for a point $Q \in \Omega$ are given by:

$$
\begin{aligned}
& c_{i k} N_{k j}^{(l)}(Q)+\int_{\Gamma} S_{k i j}^{*}(Q, P) u_{k}(P) d \Gamma(P)=\int_{\Gamma} D_{k i j}^{*}(Q, P) t_{k}^{(l)}(P) d \Gamma(P) \\
& \quad+\int_{\Omega} D_{k i j}^{*}(P, Q) N_{k, l l}^{(n l)}(P) d \Omega
\end{aligned}
$$

where $D_{i k j}$ and $S_{i k j}$ are linear combinations of plane elasticity fundamental solutions.

The integral equation for anisotropic thin plate, obtained by the reciprocity and Green theorems, and by equation (5), is given by:

$$
\begin{aligned}
K u_{3}(Q) & +\int_{\Gamma}\left[V_{n}^{*}(Q, P) w(P)-M_{n}^{*}(Q, P) \frac{\partial w(P)}{\partial n}\right] d \Gamma(P)+\sum_{i=1}^{N_{c}} R_{c_{i}}^{*}(Q, P) u_{3_{c i}}(P) \\
= & \sum_{i=1}^{N_{c}} R_{c_{i}}(P) u_{3_{c i}}^{*}(Q, P)+\int_{\Gamma}\left[V_{n}(P) u_{3}^{*}(Q, P)-M_{n}(P) \frac{\partial u_{3}^{*}}{\partial n}(Q, P)\right] d \Gamma(P) \\
& +\int_{\Omega} N_{i j}(P) u_{3, i j}^{*}(Q, P) d \Omega+\int_{\Gamma}\left[t_{i}(P) u_{3}^{*}(Q, P) u_{3, i}(P)\right. \\
& \left.-t_{i}(P) u_{3}(P) u_{3, i}^{*}(Q, P)\right] d \Gamma
\end{aligned}
$$

where $\frac{\partial()}{\partial n}$ stands for derivative in the direction of the vector $\mathbf{n}$ that is normal to the boundary $\Gamma$ in the outward direction of domain $\Omega ; M_{n}$ and $V_{n}$ are bending moment and equivalent shear force, respectively; $R_{c}$ is the thin plate corner reaction; $u_{3_{c i}}^{*}$ is the deflection of the corner $i$; constant $K$ is introduced in order to take into account that the source point $Q$ can be in the domain, on the boundary, or outside the domain. Fundamental solutions of anisotropic thin plate can be found in Albuquerque et al. [10]. 
An additional equation is necessary in order to have an equal number of unknown variables and equations. This equation is given by:

$$
\begin{aligned}
K \frac{\partial u_{3}}{\partial m}(Q)+\int_{\Gamma}\left[\frac{\partial V_{n}^{*}}{\partial m}(Q, P) w(P)-\frac{\partial M_{n}^{*}}{\partial m}(Q, P) \frac{\partial w(P)}{\partial n}\right] d \Gamma(P) \\
\quad+\sum_{i=1}^{N_{c}} \frac{\partial R_{c_{i}}^{*}}{\partial m}(Q, P) u_{3_{c i}}(P)=\sum_{i=1}^{N_{c}} R_{c_{i}}(P) \frac{\partial u_{3_{c i}}^{*}}{\partial m}(Q, P) \\
\quad+\int_{\Gamma}\left[V_{n}(P) \frac{\partial u_{3}^{*}(Q, P)}{\partial m}-m_{n}(P) \frac{\partial^{2} u_{3}^{*}}{\partial n \partial m}(Q, P)\right] d \Gamma(P) \\
\quad+\int_{\Omega} u_{3}(Q, P) N_{i j}(Q, P) \frac{\partial u_{3, i j}^{*}(Q, P)}{\partial m} d \Omega+\int_{\Gamma}\left[t_{i}(P) u_{3}^{*}(Q, P) \frac{\partial u_{3, i}(P)}{\partial m}\right. \\
\left.\quad-t_{i}(P) u_{3}(P) \frac{\partial u_{3, i}^{*}(Q, P)}{\partial m}\right] d \Gamma,
\end{aligned}
$$

where $\frac{\partial()}{\partial m}$ is the derivative in the direction of the vector $\mathbf{m}$ that is normal to the boundary $\Gamma$ on the source point $Q$ in the outward direction of the domain $\Omega, t_{i}=N_{i j} n_{j}$ and $t_{i}^{(n l)}=N_{i j}^{(n l)} n_{j}$.

As can be seen, domain integrals arise in all integral equations. These domain integrals are given in the form:

$$
P(Q)=\int_{\Omega} b(P) v^{*}(Q, P) d \Omega,
$$

where $b$ and $v^{*}$ are generic body force and fundamental solution, respectively.

The body force is approximated over the domain $\Omega$ as a sum of $M$ products between approximation functions $f_{m}$ and unknown coefficients $\gamma_{m}$, that is:

$$
b(P)=\sum_{m=1}^{M} \gamma_{m} f_{m}+a x+b y+c
$$

with

$$
\sum_{m=1}^{M} \gamma_{m} x_{m}=\sum_{m=1}^{M} \gamma_{m} y_{m}=\sum_{m=1}^{M} \gamma_{m}=0
$$

Domain integrals in the form of equation (10) approximated by the series given by (11) and (12) are transformed into boundary integrals by the radial integration method. Details of this method was given by Albuquerque and Aliabadi in [11] and will not be repeated here.

\section{NUMERICAL RESULTS}

Consider a clamped square plate, with side length $a$ and thickness $h$, subjected to a uniform transverse load $q$. This plate is discretized using 4, 8 and 16 discontinuous boundary elements of equal length and 1, 9 and 25 uniformly distributed internal points. 15, 30 and 60 load steps are used in the total incremental method. The situation analysed is quasi-isotropic $\left(E_{1} / E_{2} \cong 1\right)$. Other material constants are given by $\nu_{12}=0.316$ and $G_{12}=E_{2} /\left(1+\nu_{12}\right)$. 
Results for the combination of all the discretization parameters are shown in the figures 3, 2 and 1 together with results obtained by Purbolaksono and Aliabadi [5] for the isotropic situation. The following normalizations are applied:

$$
Q=\frac{q a^{4}}{E_{2} h^{4}}
$$

and

$$
Z=\frac{u_{3 c}}{h}
$$

where $u_{3 c}$ is the transversal displacement at the centre of the plate.
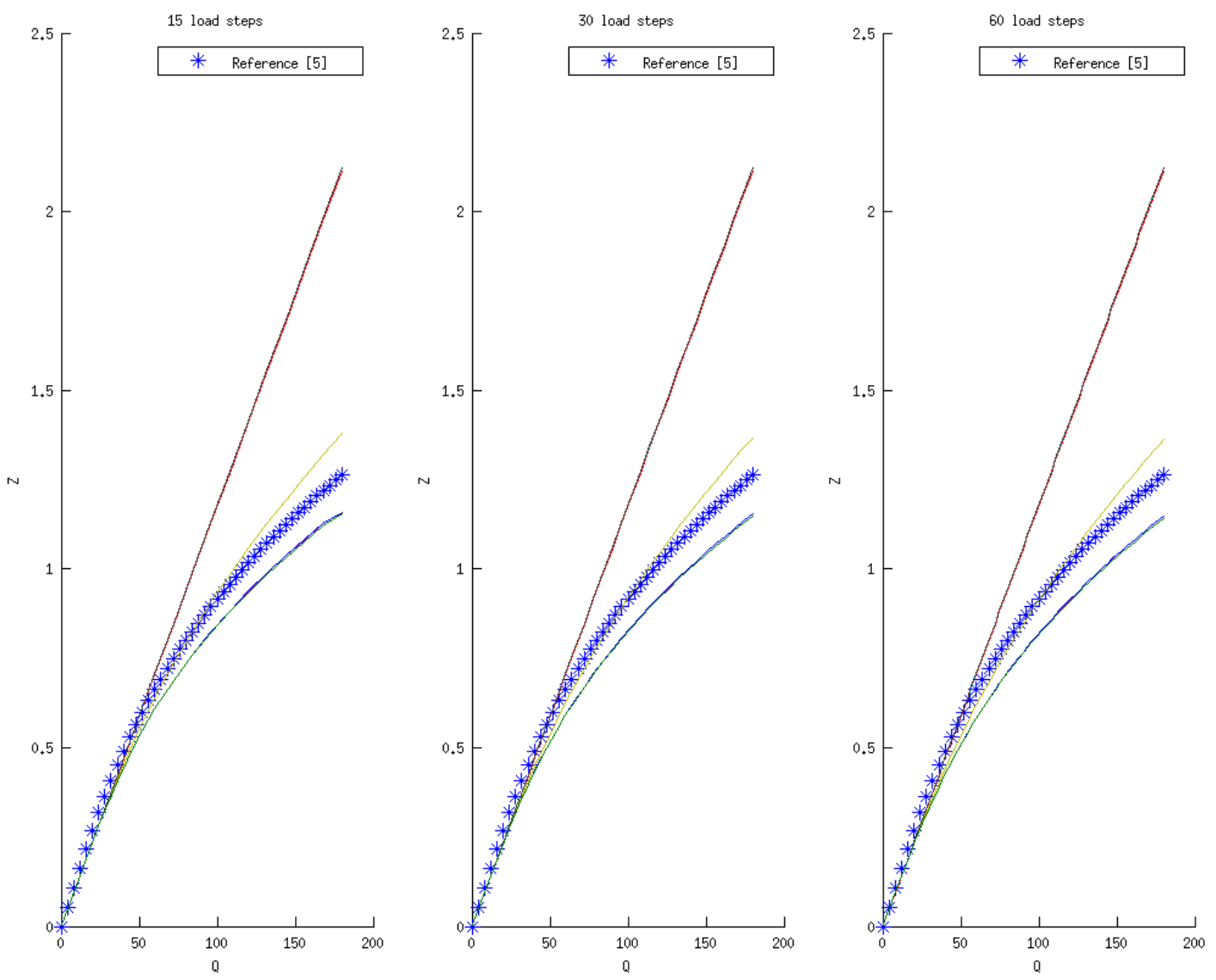

Figure 1. Normalized central deflection versus normalized load for the clamped square plate under uniformly distributed load. 

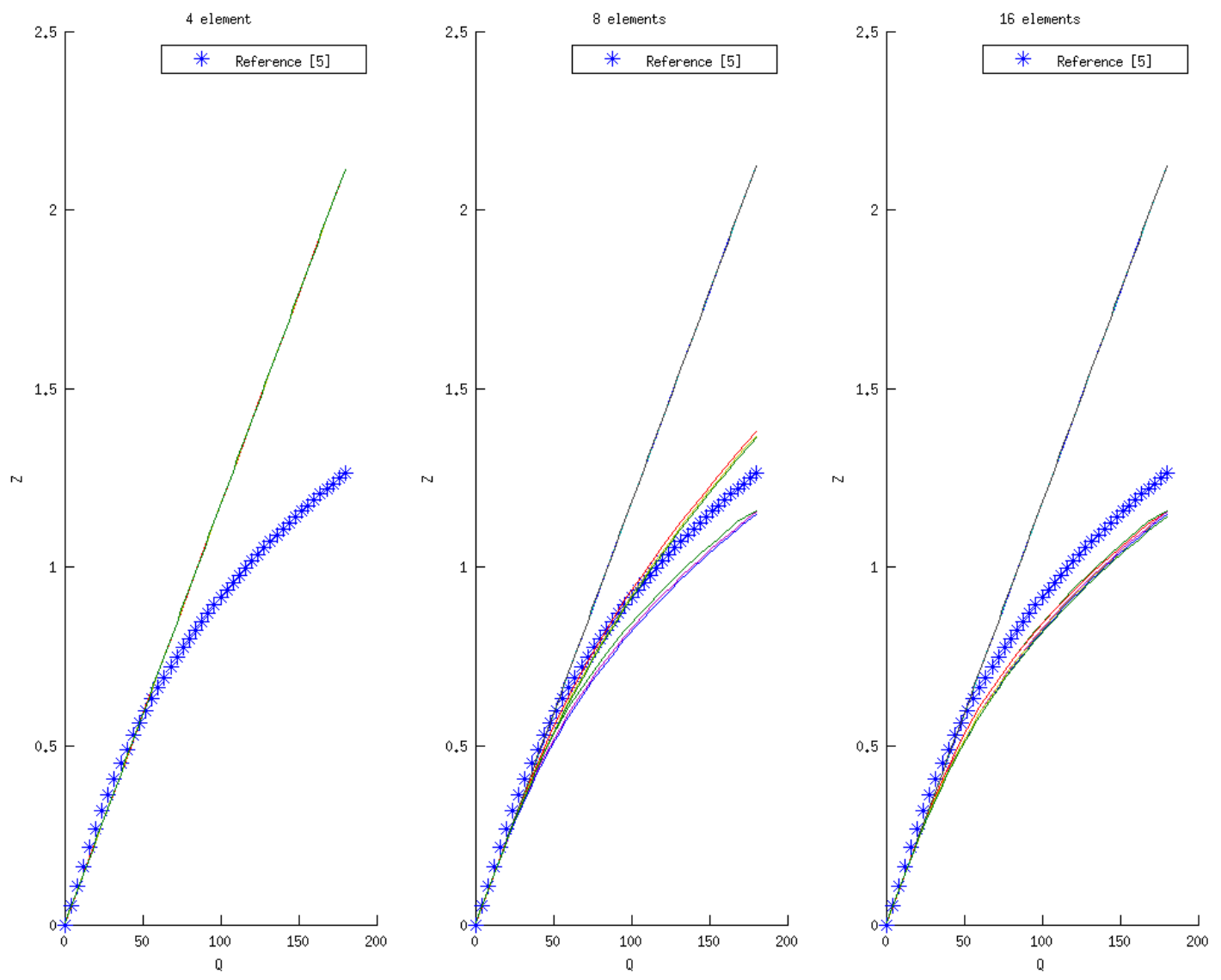

Figure 2. Normalized central deflection versus normalized load for the clamped square plate under uniformly distributed load. 

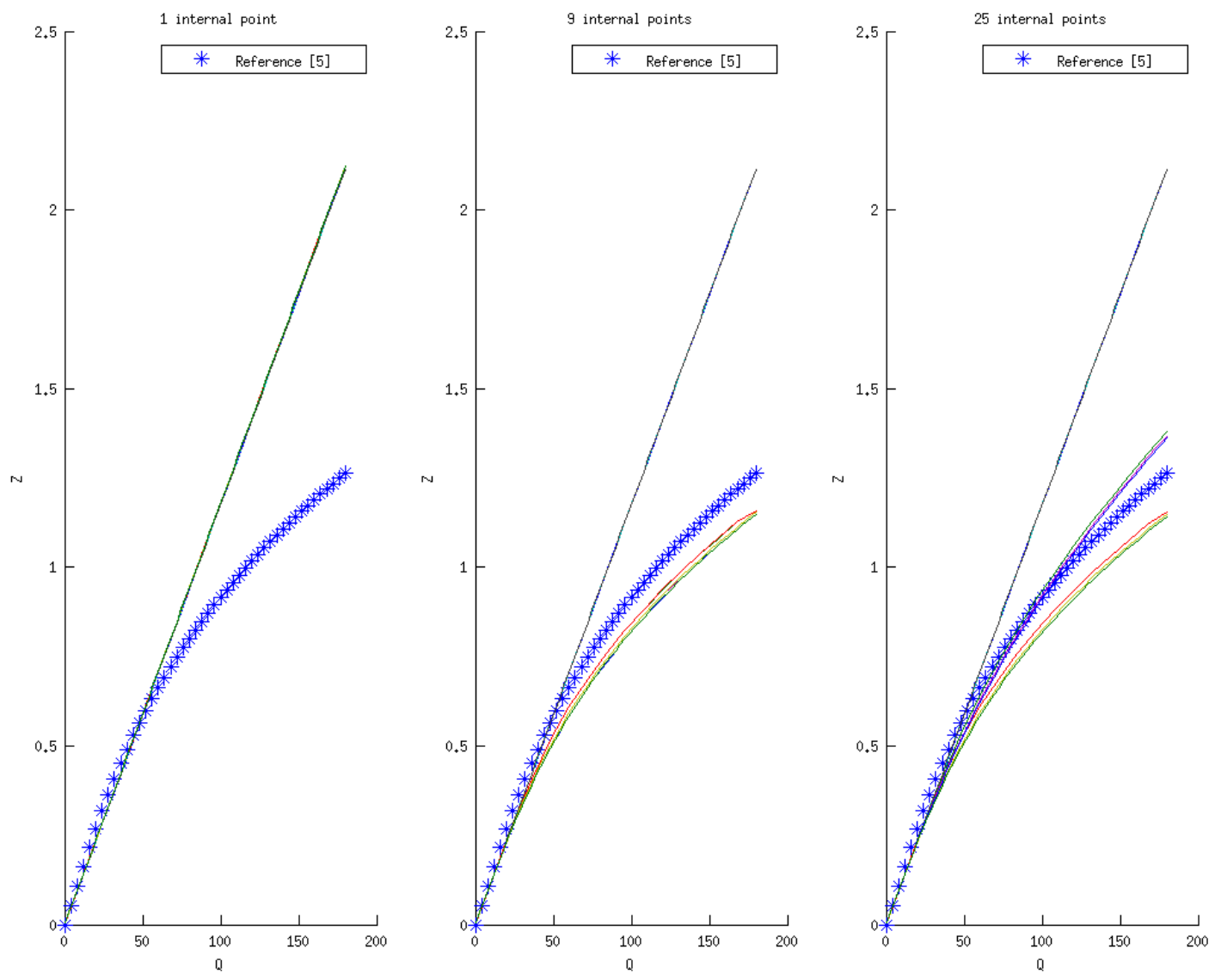

Figure 3. Normalized central deflection versus normalized load for the clamped square plate under uniformly distributed load. 
We can easily seen that for poor discretizations the non-linear effects are not taken into account and the response is linear. From Figures 3 and 1 we can conclude that only one internal and one element per side are not enough for the radial integration method to satisfactorily integrate the non-linear equations. However for superior values of discretizations, there is a good agreement between the isotropic results of Purbolaksono and Aliabadi [5] and the isotropic results obtained in this work.

\section{CONCLUSIONS}

This paper presented a boundary element formulation for the analysis of large deflections in thin plates. The formulation has a pair of coupled non-linear fourth order equations for the transverse displacement and the stress function for the in-plane stress resultants. Domain integrals that arise from non-linear terms are transformed into boundary integrals using the radial integration method. The numerical results presented good agreement with literature, which demonstrates the adaptability of the boundary element methods to non-linear problems.

\section{Acknowledgement}

The authors would like to thank P. M. Baiz and M. H. Aliabadi for the support for this work. 


\section{REFERENCES}

[1] M. H. Aliabadi. "Boundary element method, the application in solids and structures." John Wiley and Sons Ltd New York, 2002.

[2] J. Purbolaksono and M. H. Aliabadi. "Buckling analysis of shear deformable plates by boundary element method." International Journal for Numerical Methods in Engineering 62:537-563, 2005.

[3] P. H. Wen, M. H. Aliabadi, and A. Young. "Large deflection analysis of reissner plate by boundary element method." Computers and Structures 83:870â879, 2005.

[4] P. H. Wen, M. H. Aliabadi, and A. Young. "A post buckling analysis of reissner plates by the boundary element method." Journal of Strain Analysis for Engineering Design 41:239â252, 2006.

[5] J. Purbolaksono and M. H. Aliabadi. "Large deformation of shear-deformable plates by the boundary-element method." Journal of Engineering Mathematics 51:211â230, 2005.

[6] C. Y. Chia. "Nonlinear analysis of plates." MacGraw-Hill New York, 1980.

[7] G. Shi. "Flexural vibration and buckling analysis of orthotropic plates by the boundary element method." J. of Solids and Structures 26:1351â1370, 1990.

[8] P. E. OâDonoghue e S. N. Atluri. "Field/boundary element method approach to the large deflexion of thin flat plates." Computers and Structures pages 427â435, 1987.

[9] P. Sollero and M. H. Aliabadi. "Fracture mechanics analysis of anisotropic plates by the boundary element method." Int. J. of Fracture 64:269â284, 1993.

[10] E. L. Albuquerque, P. Sollero, W. Venturini, and M. H. Aliabadi. "Boundary element analysis of anisotropic kirchhoff plates." International Journal of Solids and Structures 43:4029â4046, 2006.

[11] E. L. Albuquerque and M. H. Aliabadi. "A boundary element formulation for boundary only analysis of thin shallow shells." CMES - Computer Modeling in Engineering and Sciences 29:63â73, 2008. 